\title{
Revealing spatio-temporal patterns of rabies spread among various categories of animals in the Republic of Kazakhstan, 2010-2013
}

\author{
Sarsenbay K. Abdrakhmanov, ${ }^{1}$ Kanatzhan K. Beisembayev, ${ }^{1}$ Fedor I. Korennoy, ${ }^{2}$ \\ Gulzhan N. Yessembekova, ${ }^{1}$ Dosym B. Kushubaev, ${ }^{1}$ Ablaikhan S. Kadyrov ${ }^{1}$ \\ 'S. Seifullin Kazakh Agro-Technical University, Astana, Kazakhstan; \\ ${ }^{2}$ Federal Center for Animal Health (FGBI ARRIAH), Vladimir, Russia
}

\begin{abstract}
This study estimated the basic reproductive ratio of rabies at the population level in wild animals (foxes), farm animals (cattle, camels, horses, sheep) and what we classified as domestic animals (cats, dogs) in the Republic of Kazakhstan (RK). It also aimed at forecasting the possible number of new outbreaks in case of emergence of the disease in new territories. We considered cases of rabies in animals in RK from 2010 to 2013, recorded by regional veterinary services. Statistically significant space-time clusters of outbreaks in three subpopulations were detected by means of Kulldorff Scan statistics. Theoretical curves were then fitted to epidemiological data within each cluster assuming exponential initial growth, which was followed up by calculation of the basic reproductive ratio $R_{0}$. For farm animals, the value of $\mathrm{R}_{0}$ was 1.62 (1.11-2.26) and for wild animals 1.84 (1.083.13 ), while it was close to 1 for domestic animals. Using the values obtained, an initial phase of possible epidemic was simulated in order to predict the expected number of secondary cases if the disease were introduced into a new area. The possible number of new cases for 20 weeks was estimated at 5 (1-16) for farm animals, 17 (1-113) for wild animals and about 1 in the category of domestic animals. These results
\end{abstract}

Correspondence: Sarsenbay K. Abdrakhmanov, S. Seifullin Kazakh AgroTechnical University, 62 av. Pobeda, 010011 Astana, Kazakhstan.

Tel. + 77.013.881467.

E-mail: S_abdrakhmanov@mail.ru

Key words: Kazakhstan; Rabies; Spatio-temporal patterns; GIS.

Acknowledgements: this work was accomplished under a Budgetary Program of Ministry of Agriculture of the Republic of Kazakhstan \#0115 RK01952 Scientific support of veterinary well-being.

Received for publication: 23 January 2016.

Revision received: 10 February 2016.

Accepted for publication: 13 February 2016.

CCopyright S.K. Abdrakhmanov et al., 2016

Licensee PAGEPress, Italy

Geospatial Health 2016; 11:455

doi:10.4081/gh.2016.455

This article is distributed under the terms of the Creative Commons Attribution Noncommercial License (CC BY-NC 4.0) which permits any noncommercial use, distribution, and reproduction in any medium, provided the original author(s) and source are credited. have been used to produce set of recommendations for organising of preventive and contra-epizootic measures against rabies expected to be applied by state veterinarian services.

\section{Introduction}

The epidemic situation with respect to rabies in the endemic countries in the world, including the Republic of Kazakhstan (RK), is characterised by an uneven spread of infection. The disease is registered on every continent except Australia and Antarctica (Smreczak et al., 2012; Youla et al., 2014; Eckardt et al., 2015). The disease has an acute course with overt signs of polyencephalomyelitis and the mortality is $100 \%$ in the absence of immediate treatment. Due to the accession of RK to the World Trade Organization (WTO), the need for socio-economic stability, including epizootics that may extend to humans, is increasingly important for each administrative unit of the country. Currently, an ascending trend of rabies with an average increase of 7\% per year among susceptible animals (fox, raccoon dogs, wolves, cats and cattle) is being observed in RK. About 700 heads of farm animals perish annually from rabies in the republic and more than $50 \%$ of them consist of cattle and up to $25 \%$ of small ruminants (Makarov et al., 2008; Abdrakhmanov et al., 2010). Rabies gets the attention of the veterinary services, and mass vaccination campaigns and surveillance are applied both in the population of wild animals as well as in livestock and other domestic animals. Predicting the size of possible epidemics in the event of new rabies outbreaks is one of the priorities of the veterinary service. The number of recorded cases in different animal categories during 2010-2013 was studied in order to identify the main epidemiological patterns of the disease at the population level. During this study, significant spatio-temporal clusters of the disease were identified; epidemic curves built for each cluster and the values of the basic reproductive ratio $\left(\mathrm{R}_{0}\right)$ for each sub-population calculated (Iglesias et al., 2011, 2015). The values obtained were then used to simulate the possible number of new outbreaks over a period of 20 weeks in the case of a new infection focus emergence.

\section{Materials and Methods}

\section{Study area}

The whole territory of RK, covering an area of 2,724,902 $\mathrm{km}^{2}$, was used as a study area in this research. RK is $9^{\text {th }}$ largest country in the world and $4^{\text {th }}$ largest in Eurasia with total population of more than 17 billion. The first-level administrative division is represented by 14 regions 
(oblasts), the spatial extent of which ranges from 117,249 to $427,982 \mathrm{~km}^{2}$ (Figure 1). Agriculture, especially cattle breeding, is one of the priority sectors of the economics of RK. As of January 2015, the number of cattle (in thousands of heads) reached 6028.0, sheep - 15,532.4, goats 2378.9, pigs -844.2 , horses -1936.7 and camels -165.9 .

\section{Data}

The data on rabies outbreaks in RK during 2010-2013 were provided by the national veterinary service and recorded during expedition trips. The database consists of 496 outbreaks in animals such as cats, dogs, cows, foxes, camels, sheep and horses (Table 1). To facilitate modelling, all kinds of animals were divided into three categories: farm animals (cattle, camels, horses, sheep) domestic animals (cats and dogs) and wild animals that includes only foxes. Among the total number of outbreaks, $60 \%$ were in farm animals (296 outbreaks), 13\% in domestic animals (69 outbreaks), and $27 \%$ in wild animals (131 outbreaks).

Rabies outbreaks have the following attributes, which are relevant for further analysis: geographic coordinates (latitude, longitude); date of the outbreak; number and type of infected animals. The data used in this study were presented in the format of Microsoft Excel spreadsheet, and converted to ESRI shapefiles (http://www.esri.com/) for cartographic representation. Figure 1 shows a map of RK overlaid with cases of rabies in the three sub-populations mentioned above.

\section{Methods}

The first stage of the work was to identify space-time clusters of outbreaks in each of the three categories by means of Kulldorff Scan statistics (Kulldorff et al., 2005). The space-time permutations type of analysis was chosen. The preliminary analysis of the data using the software tool Multi-distance Spatial Cluster Analysis (Ripley's K-function) was performed in order to determine the maximum distance of spatial outbreak clustering, which gives an idea of the maximum spa-

Table 1. List of animals registered as infected by rabies in the 2010-2013 period.

\begin{tabular}{lc} 
Species & Infected animals (n) \\
Camel & 10 \\
Cat & 1 \\
\hline Cow & 237 \\
Dog & 68 \\
\hline Fox & 127 \\
Horse & 24 \\
\hline Sheep & 29
\end{tabular}

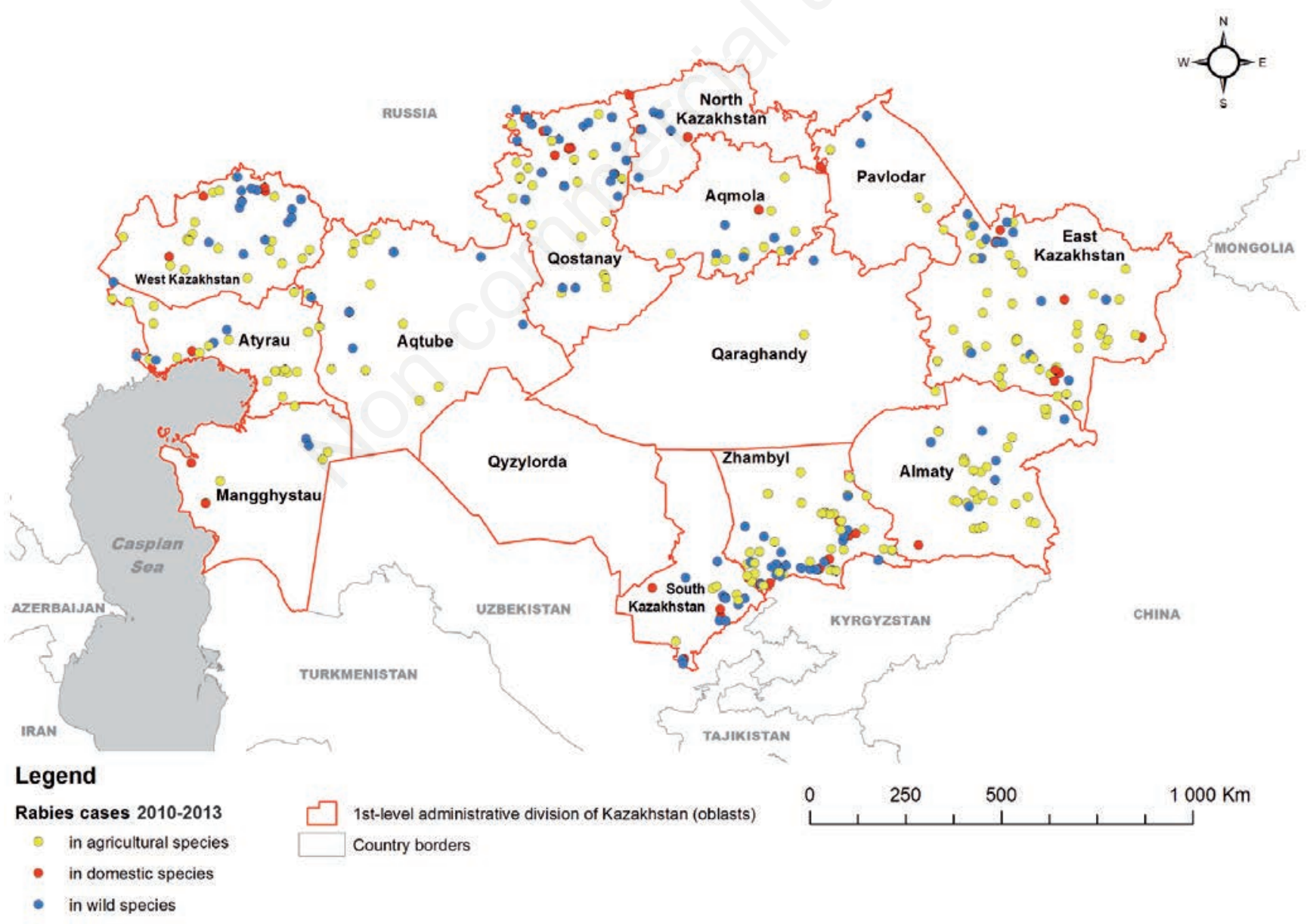

Figure 1. Geographical location of the Republic of Kazakhstan and its first-level administrative divisions with reported rabies cases in animals, 2010-2013. 
tial cluster size (Mitchell, 2005). After detection of the spatio-temporal clusters, only those that were statistically significant at $\mathrm{P} \leq 0.05$ ), and where the number of outbreaks were sufficiently numerous for the construction of an epidemic curve (at least seven outbreaks), were selected for further analysis. This curve was constructed for each of the selected clusters based on the available data about the dynamics of rabies cases at the second stage of the analysis. Time step of the epidemic curve implies a period equal to the average incubation period of rabies for the respective category of animal. This is based on the assumption that the animal may already be a source of infection for other susceptible animals during the incubation period, and hence the duration of the incubation period can be taken as the duration of infectivity. Literature data on rabies (Wunner and Jackson, 2010; Alabama Department of Public Health, 2015) suggest that the duration of incubation period may be: 210 weeks for cattle, but may take up to 6 months; and 3-8 weeks usually for dogs, but can last for up to 8.5 months. The duration of the infectious period for foxes was taken to be similar to that in dogs. Based on these ranges, durations of the infectious periods (D) were modelled using Pert distribution (Van Hauwermeiren and Vose, 2009) with the following parameters: for cattle (farm animals) - Pert (0; 7.3; 25); for dogs, cats and foxes (domestic and wild animals) - Pert ( $0 ; 4.5 ; 36)$.

This would allow accounting for uncertainties in the duration of the infectious period and getting the mean values and limits of $95 \%$ confidence intervals (CI) during the further analysis. The means for the two above distributions amounted to 9 weeks. This value was used as a time step ( $t$ ) for the epidemic curves.

In order to model the possible number of new rabies outbreaks in the event of an epidemic in a new territory, the simplest technique based on the concept of basic reproductive ratio was applied. This approach refers to the analysis of the epidemic curve and assumes exponential growth of the number of new cases since the beginning of the epidemic until its maximum (peak) (Dietz, 1993; Heffernan et al., 2005). The key concept of this approach is the basic reproductive ratio $\left(\mathrm{R}_{0}\right)$, which shows the average number of secondary infections, which can be caused by one infected individual during its infectious period. The formula for calculating $R_{0}$ from epidemiological data is as follows:

$$
R_{0}=1+\frac{D}{\Delta t} \ln \left(\frac{N_{2}}{N_{1}}\right)
$$

where: $D$ is the duration of the infectious period, i.e. the period of time during which the infected individual (or herd) can be a source of infection for other susceptible animals (herds); $t$ is time interval between two observations at which $N_{l}$ and $N_{2}$ outbreaks were recorded. $t$ also acts as a time step of epidemic curve.

Values of $R_{0}$ greater than 1 usually indicate an increase of an epidemic, while values less than 1 show an attenuation. $R_{0}=1$ indicates creeping endemic course of the disease without a sharp rise. Thus,

\section{Legend \\ 1-level administrative units of Kazakhstan ("oblasts") \\ - rabies cases in agricultural (farm) species, cluster \# 6}

Figure 2. Statistically significant cluster of rabies cases in farm species. 
knowing the value $R_{0}$, the possible number of new outbreaks may be estimated by the inverse formula:

$$
N_{2}=N_{1} \operatorname{Exp}\left(\frac{\left(R_{0}-1\right) \Delta t}{D}\right)
$$

In addition, knowledge of the basic reproductive ratio $\left(\mathrm{R}_{0}\right)$ enables the calculation of the herd immunity threshold $\left(H_{t}\right)$, i.e. the share of a susceptible livestock that should be vaccinated (alternatively, disposed of) to prevent an epidemic. According to Anderson and May (1992), $\mathrm{H}_{\mathrm{t}}$ can be calculated using the formula:

$$
H_{t}=1-\frac{1}{R_{0}}
$$

After building the epidemic curve for each cluster, approximating exponential curves were fitted to initial segments of the curves (phases of growth) by means of the least square method (Wolberg, 2006), after which $R_{0}$ values were calculated by equation 1 . Accounting for uncertainty in duration of infectious period $D$, expressed by the distributions, allowed estimating the average values of $\mathrm{R}_{0}$ and the limits of $\mathrm{CI}$ 95\%. Modelling was performed using the method of Monte Carlo simulations at 10,000 iterations (Vose, 2008). Then, simulation of the possible number of new rabies cases was performed using the obtained values $R_{0}$ for a time period of 20 weeks by means of equation 2 . The herd immunity threshold $H_{t}$ was calculated using equation 3 , which in the case of rabies shows the proportion of susceptible population that must be vaccinated to prevent an epidemic development.

\section{Software}

Geospatial analysis, data processing and visualisation were performed using geographic information system ArcGIS 10.3.1 (http://www.esri.com/). Identification of space-time clusters was performed by means of SatScan 9.1 software package (Kulldorff, 2015). Construction of epidemic curves, fitting of approximating exponential curves, and simulation by the Monte Carlo method were performed using the software package @Risk 6.2 (http://www.palisade.com/risk/) based on Microsoft Excel.

\section{Results}

\section{Cluster detection}

The analysis of rabies cases during 2010-2013 using Multi-distance Spatial Cluster Analysis (Ripley's K-function) procedure showed that the maximum distance, at which grouping of cases can be observed is about $500 \mathrm{~km}$. Therefore, a maximum search radius of $250 \mathrm{~km}$ was adopted to identify potential clusters. In so doing only one statistically significant cluster that also had a sufficient number of outbreaks for the construction of an epidemic curve was revealed for each sub-population of animals. These clusters are represented in the maps in Figures 2-4.

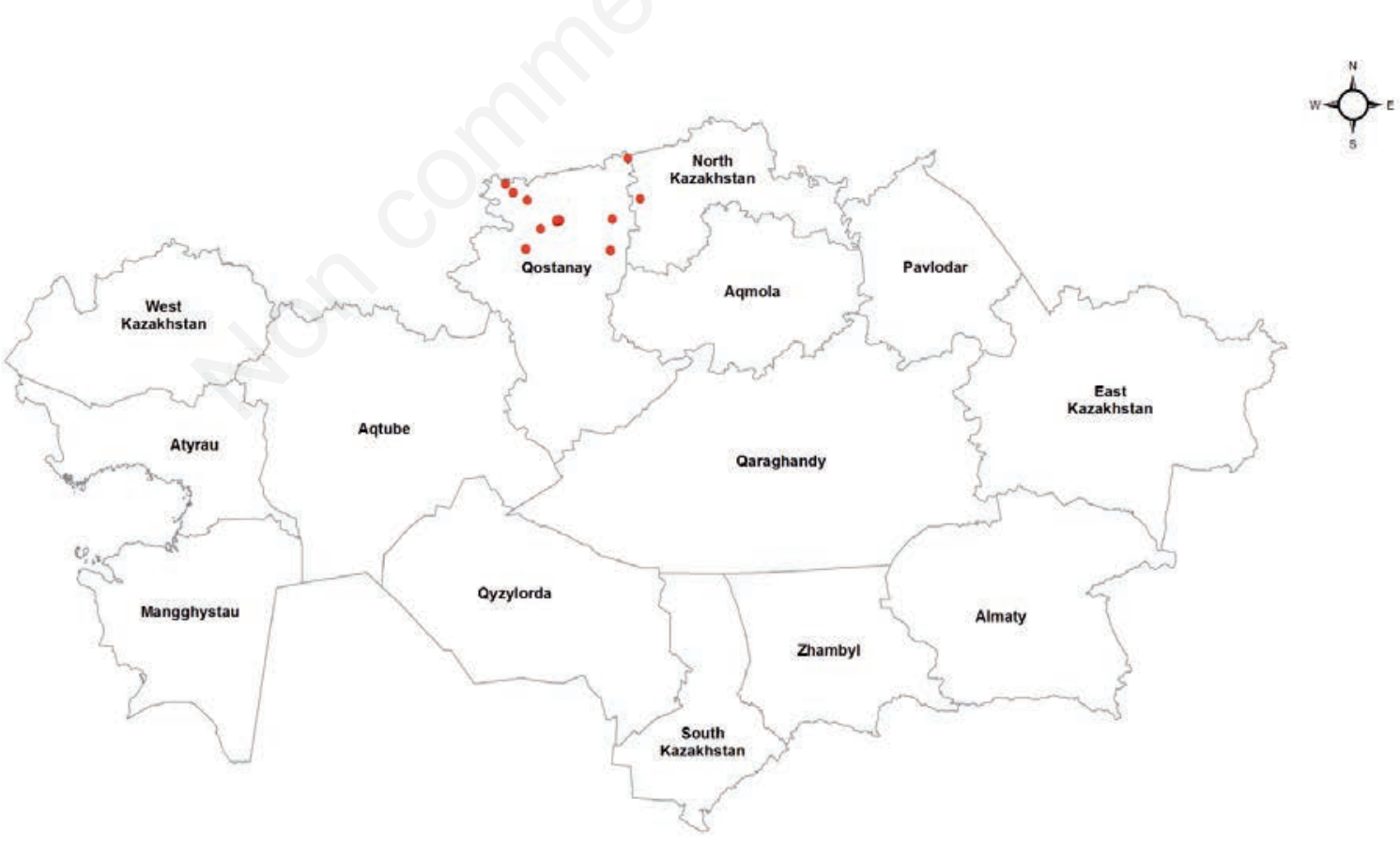

\section{Legend}

1-level administrative units of Kazakhstan ("oblasts")

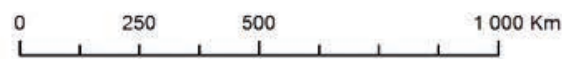

- rabies cases in domestic species, cluster \# 2

Figure 3. Statistically significant cluster of rabies cases in domestic species. 
In the category of farm animals, the clusters were mainly found among cattle, while in the category of domestic animals, it was found among dogs. Clusters representing foxes (the wild animal category) were also observed. The initial segments of epidemic curves plotted for the three identified clusters, including fitted exponential curves, are shown in Figures 5-7.

\section{The basic reproductive ratio}

The following values were obtained by calculation of the basic reproductive ratio: $\mathrm{R}_{0}=1.62$ (1.11-2.26) for farm animals; $\mathrm{R}_{0}=1.84$ (1.08-3.13) for foxes (wild animals); $R_{0}=$ close to 1 for domestic animals with no significant growth seen in the available data.

These values confirm the hypothesis of the secondary character of epidemics in the domestic animals since, apparently, epidemics in these animal populations do not tend to develop independently but are supported by contact with wild animals.

\section{Modelling of epidemic size}

Taking into account the values obtained for the basic reproductive ratio $\mathrm{R}_{0}$, modelling of the possible number of outbreaks, according to equation 2, in each of the three categories of animals for a period of 20 weeks demonstrated the following results: 5 (1-16) new outbreaks may occur among the farm animals; 1 outbreak could occur among the domestic animals but there is a large uncertainty, because their number is obviously highly dependent on the presence of outbreaks among the wild animals; 17 (1-113) new cases of rabies may occur among the wild animals.

\section{Calculation of the required proportion of vaccination}

Calculation of herd immunity threshold using equation 3 gave the following values of the proportion of livestock that would need to be vaccinated to prevent an epidemic development: $36 \%$ (10-56\%) of livestock should be vaccinated in the category of farm animals; $41 \%$ (7$68 \%$ ) of livestock should be vaccinated in the category of wild animals; an adequate calculation of the herd immunity threshold for the domestic animals was not possible due to the proximity of $R_{0}$ to 1 .

\section{Discussion}

According to the World Health Organization (WHO), rabies ranks fifth among all infectious diseases in terms of economic effects (WHO, 2015). Rabies occupies a special place among the variety of zoonotic infectious diseases because this virus affects almost all warm-blooded animals along with humans. Therefore, the problem of rabies must be jointly studied by medical and veterinary professionals. Indeed, the risk of the disease spreading among animals and humans has not decreased in RK in recent years. Activation of natural foci of rabies is periodically seen in almost all regions of the country, with a growing number of cases among wild carnivores and domestic animals as well as farm animals.

Some authors have noted a certain correlation of rabies registration among farm livestock and domestic carnivores during studies of interspecies transfer between key populations of susceptible animals, thus suggesting a possible risk not only among wild animals but also of man-

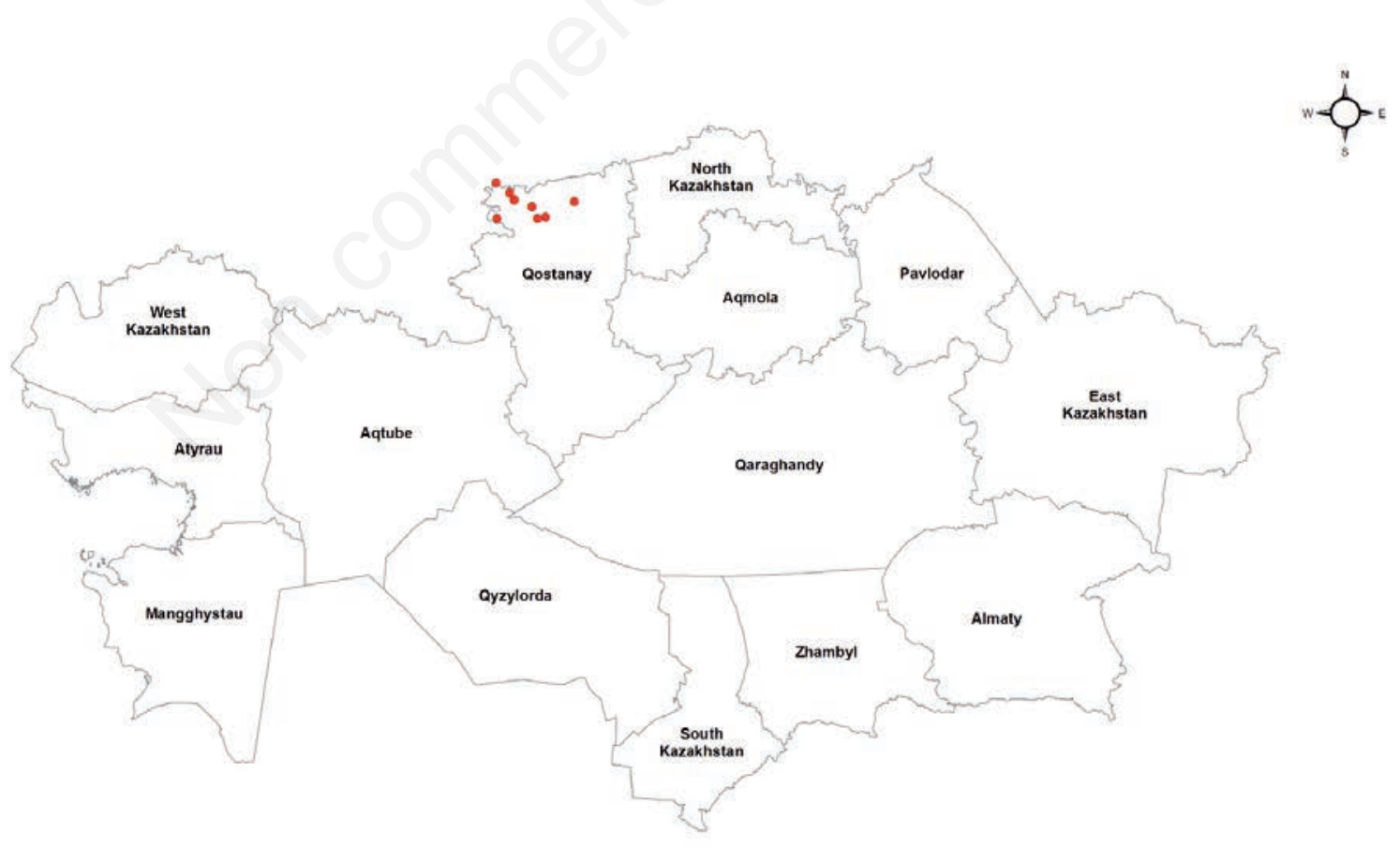

\section{Legend}

1-level administrative units of Kazakhstan ("oblasts")

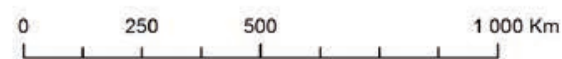

- rabies cases in wild species, cluster \#2

Figure 4. Statistically significant cluster of rabies cases in wild species. 
ifestation of an urban type of rabies (Shestopalov et al., 2001; Abdrakhmanov et al., 2010; Abdrakhmanov and Eseneva, 2012). The analysis shows the presence of the positive dynamics of rabies epidemics in the categories of farm animals and wild animals. An epidemic development, with an increase in the number of infected animals (herds), is possible in these populations in the event of new outbreaks within high-risk areas. Rabies in the population of dogs and cats is apparently supported only as a result of contact with a wild animal population, and the epidemic would decay in the absence of such contacts. The epidemic curves plotted for the three identified clusters and the fitted exponential curves (Figures 5-7) confirm the hypothesis of the secondary character of epidemics in domestic animals since these animals are thought to have been infected by contact with wild animals. The values found agree well with the results of other authors (Hampson et al., 2009; Zinsstag et al., 2009).

Active manifestations of an epidemic process of the same infectious disease in different areas (farms, settlements and administrative areas, etc.) will always vary due to exposure to various anthropogenic and biogenic factors. The basic and additional evaluation criteria are present in each disease, the nature and intensity of which determines the growth or fading of any potential epidemic process.

Given the basic reproductive ratio of rabies epidemic process in RK in three of the most significant animal populations, an attempt was made to determine the appropriate proportion of animals needing vaccination, which is the main, general measure to combat rabies. Our results show that, on average, $36 \%$ of the animals should be vaccinated in the farm animal category, while the figure increased to $41 \%$ for the category of wild animals. The resulting ratio of vaccinations among the productive and wild carnivores (the main natural reservoir) corresponds to the current vaccination in the country. As for domestic carnivores, the situation is different: with $\mathrm{R}_{0}$ equal or close to 1 , the disease situation has the character of endemicity without epidemic manifestations. This outcome could be due to the reduction in the number of stray carnivores and successful control of the population within the period subjected to the analysis (2010-2013).

The geographical overlap of the clusters of domestic and wild animals (Figures 3 and 4), as well as the matching time period (SpringAutumn 2010), indicate the possibility for mutual contamination of the two populations. The proximity of the two clusters to the national border should be noted, as it can be interpreted as a possible transboundary transfer of rabies in the population of wild animals with further spread into the population of domestic animals.

It should be noted that the only statistically significant spatio-temporal cluster of rabies in the population of farm animals was detected in the East Kazakhstan region, where the highest population of susceptible species was recorded (about 870 thousand heads compared to 300 400 thousand heads in other areas as of 2014) according to the Veterinary Service of RK. This can be seen as an indication that the potentially most likely rabies epidemic would develop in high-density populations of susceptible livestock. In areas with relatively low population densities, only occasional pockets of rabies usually arise and they do not lead to sharp increases of the number of cases. In this regard, our analysis can be considered a worst-case scenario, and the predicted number of possible outbreaks reflects a highest possible outcome of a potential epidemic.

The continuing unfavourable epidemic situation with regard to rabies in the country is due to the widespread of feral rabies, poor performance on the regulation of the number of wild animals and organisation of oral immunisation, the increase of number of stray animals in urban and rural areas, gross violations of farm animals housing, poor organisation of accounting and registration as well as insufficient public awareness campaign among the human population. Further work on the development of the model used may rely on the creation of an integrated model of rabies epidemic process, including the interaction between different populations, and accounting for data on vaccination. The results obtained have been used to compile a set of methodological recommendations regarding the organisation of preventive and contra-epizootic measures against rabies, which are expected to be applied by state veterinarian services.

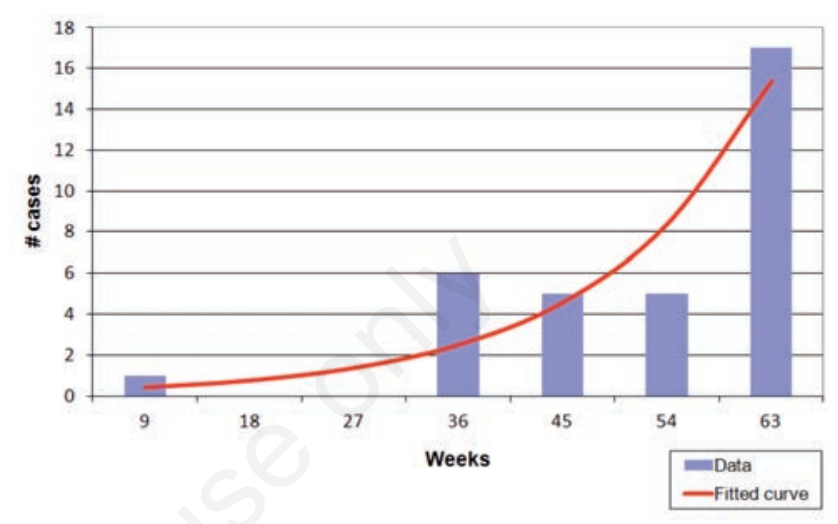

Figure 5. Epidemic data with fitted exponential curve for statistically significant cluster of rabies cases in farm species.

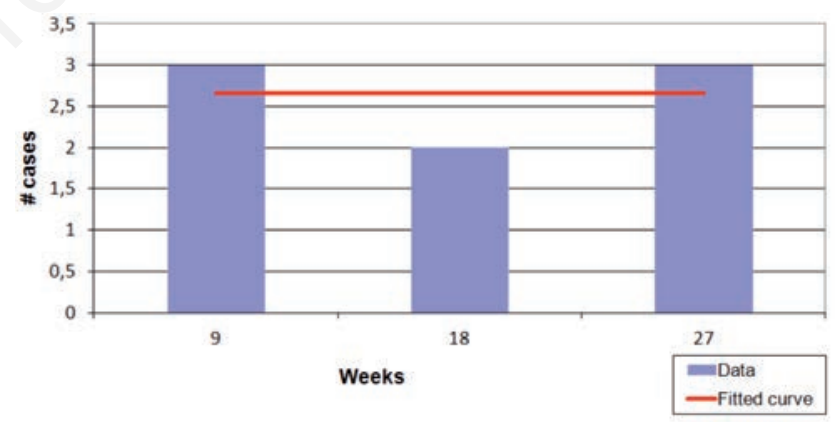

Figure 6. Epidemic data with fitted exponential curve for statistically significant cluster of rabies cases in domestic species.

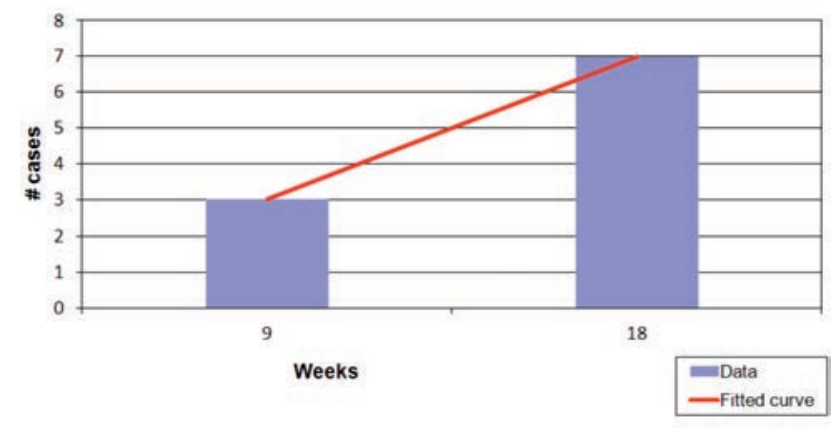

Figure 7. Epidemic data with fitted exponential curve for statistically significant cluster of rabies cases in wild species. 


\section{Conclusions}

Our study allows the estimation of basic reproductive ratio $R_{0}$ for rabies in different animals at the population level. The results demonstrate that: i) $R_{0}$ can be higher than 1 resulting in the epidemic growth in farm livestock if there is a high density of animals in the susceptible population; and ii) $\mathrm{R}_{0}$ can be higher than 1 in the population of wild carnivores, together with geographical overlap of outbreak clusters in wild and domestic species, thus indicating close interaction among epidemic processes in these sub-populations with possibilities of transfer of the disease from wild to domestic animals. Therefore, the forecast of possible size of a new epidemic should be considered a worst-case scenario when there is a high density of susceptible animals in the populations.

\section{References}

Abdrakhmanov SK, Eseneva SS, 2012. The role of various animal species in the epizootic process of rabies. In: Proceedings of International Conference Problems of control of dangerous, exotic and zooanthroponosis diseases of animals dedicated to 70-years jubilee of professor N.G.Asanov, 1:23-8.

Abdrakhmanov SK, Sytnic II, Tursunkulov SZh, 2010. Visualization and analysis of veterinary and geographical rabies spread by using GIS technologies. In: Proceedings of the 5th International Scientific Practical Conference, 2010 March 17-18, Barnaul. AGAU Publ., Barnaul, Russia, pp 283-6.

Alabama Department of Public Health, 2015. Management of animals that bite humans. Available from: http://www.adph.org/epi/assets/ MgmtAnimalBite15.pdf

Anderson RM, May RM, 1992. Infectious diseases of humans. Dynamics and control. Oxford University Press, Oxford, UK.

Dietz K, 1993. The estimation of the basic reproduction number for infectious diseases. Stat Methods Med Res 2:23-41.

Eckardt M, Freuling C, Muller T, Selhorst T, 2015. Spatio-temporal analysis of fox rabies cases in Germany 2005-2006. Geospat Health 10:313.

Hampson K, Dushoff J, Cleaveland S, Haydon DT, Kaare M, Packer C, Dobson A, 2009. Transmission dynamics and prospects for the elimination of canine rabies. PLoS Biology 7:3.

Heffernan JM, Smith RJ, Wahl LM, 2005. Perspectives on the basic reproductive ratio. J Roy Soc Interface 2:281-93.

Iglesias I, Munoz MJ, Montes F, Perez A, Gogin A, Kolbasov D, de la Torre A, 2015. Reproductive ratio for the local spread of African swine fever in wild boars in the Russian Federation. Transboundary Emerg Dis 10.1111/tbed.12337.

Iglesias I, Perez AM, Sanchez-Vizcaino JM, Munoz MJ, Martinez M, De La Torre A, 2011. Reproductive ratio for the local spread of highly pathogenic avian influenza in wild bird populations of Europe, 2005-2008. Epidemiol Infect 139:99-104.

Kulldorff M, 2015. Information management services, Inc. SatScan ${ }^{\mathrm{TM}}$ v.9.1: software for the spatial and space-time scan statistics. Available from: http://www.satscan.org/

Kulldorff M, Heffeman R, Hartman J, Assuncao RM, Mostashari FA, 2005. A space-time permutation scan statistic for the early detection of disease outbreaks. PLoS Medicine 2:216- 24.

Makarov VV, Sukhareva OI, Gulyukin AM, Litvinov OB, 2008. The trend of rabies spread in Eastern Europe. Vet Med 7:20-2.

Mitchell A, 2005. The ESRI guide to GIS analysis. Vol. 2. ESRI Press, Redlands, CA, USA.

Shestopalov AM, Kissurina MI, Gruzdev KN, 2001. Rabies and its distribution in the world. Problems of virology. 2:7-12.

Smreczak M, Orłowska A, Trębas P, Żmudziński JF, 2012. Rabies epidemiological situation in Poland in 2009 and 2010. Bull Vet Inst Pulawy 56:115-266.

Van Hauwermeiren M, Vose D, 2009. A compendium of distributions. Vose Software, Ghent, Belgium.

Vose D, 2008. Risk analysis: a quantitative guide. $3^{\text {rd }}$ ed. Wiley, Hoboken, NJ, USA.

WHO, 2015. Rabies. Available from http://www.who.int/rabies/en/

Wolberg J, 2006. Data analysis using the method of least squares. Springer-Verlag, Berlin-Heidelberg, Germany.

Wunner WH, Jackson AC, 2010. Rabies: scientific basis of the disease and its management. Academic Press, San Diego, CA, USA.

Youla AS, Traore FA, Sako FB, Feda RM, Emeric MA, 2014. Canine and human rabies in Conakry: epidemiology and preventive aspects. Bull Soc Pathol Exot 7:19-21.

Zinsstag J, Durr S, Penny MA, Mindekem R, Roth F, Menendez Gonzalez S, Naissengar S, Hattendorf J, 2009. Transmission dynamics and economics of rabies control in dogs and humans in an African city. P Natl Acad Sci USA 106:35. 